\title{
On the Expressiveness of Linearity vs Persistence in the Asychronous Pi-Calculus
}

\author{
Catuscia Palamidessi \\ INRIA and LIX École Polytechnique \\ catuscia@lix.polytechnique.fr \\ Frank D. Valencia \\ CNRS and LIX École Polytechnique \\ frank.valencia@lix.polytechnique.fr
}

\author{
Vijay Saraswat \\ IBM TJ Watson Research Lab \\ vijay@saraswat.org \\ Björn Victor \\ Dept. of Information Technology, Uppsala Univ. \\ bjorn.victor@it.uu.se
}

\begin{abstract}
We present an expressiveness study of linearity and persistence of processes. We choose the $\pi$-calculus, one of the main representatives of process calculi, as a framework to conduct our study. We consider four fragments of the $\pi$-calculus. Each one singles out a natural source of linearity/persistence also present in other frameworks such as Concurrent Constraint Programming (CCP), Linear CCP, and several calculi for security. The study is presented by providing (or proving the non-existence of) encodings among the fragments, a processes-as-formulae interpretation and a reduction from Minsky machines.
\end{abstract}

\section{Introduction}

Several process calculi such as CCS, CSP, the $\pi$-calculus [15] and Linear CCP $[8,23]$ have an obvious source of linearity: Messages (or senders) are consumed upon being received. For example, in the $\pi$-calculus, the system

$$
\bar{x}\langle z\rangle|x(y) . P| x(y) \cdot Q
$$

represents a message with a datum $z$, tagged with $x$, that can be consumed by either $x(y) . P$ or $x(y) . Q$. The system can evolve into either (a) $P\{z / y\} \mid x(y) \cdot Q$ or (b) $x(y) . P \mid Q\{z / y\}$.

Nevertheless, there are other process calculi which follow a different pattern: Messages cannot be consumed; they are persistent rather than linear. One of the most prominent representatives of such calculi is Concurrent Constraint Programming (CCP) [22]. In this framework all messages, more precisely items of information, are accumulated in a global store. The messages in the store can be read but, unlike in Linear CCP, they cannot be consumed, i.e., the store is persistent.
Several other frameworks using a persistent store can be found in the context of calculi for analyzing and describing security protocols. For instance, Crazzolara and Winskel's SPL [7], the Spi Calculus variants by Fiore and Abadi [9] and by Amadio et al [1], and the calculus of Boreale and Buscemi [4] are all operationally defined in terms of configurations containing items of information (messages) which cannot be consumed during evolution. The idea is that the persistent store models an attacker's ability to see and remember every message that has been in transit.

A legitimate question is whether such persistence restricts the systems that we can specify, model or reason about in the framework. For instance, whether CCP can specify the kind of systems that can be described in Linear CCP. Analogously, in the context of the above-mentioned calculi for security, e.g. in SPL, one may wonder if not allowing the attacker to remove messages from the network may rule out the specification of a possible attack to a given protocol. (Note that the claims of extra expressivity of Linear CCP over CCP in $[2,8]$ are based on discrimination introduced by divergence that is ignored by the standard notion of weak bisimulation.)

There is another source of linearity in (1): Receivers can also be consumed. For example, in the case in which $x(y) . P$ evolves into $P\{z / y\}$. Persistent receivers arise, e.g. in the notion of omega receptiveness [20] where the input of a name is always available - but always with the same continuation. In the $\pi$-calculus persistent receivers are used, for instance, to model functions, objects, higher-order communications, or procedure definitions. Notice that the situation in this case is somehow dual to the persistent outputs case and begs the same kind of question: If we require inputs to be persistent, do we restrict the kind of systems we can specify?

Now, in the above situations we have that either messages or receivers are persistent. One can further consider the complementary case in which both messages and re- 
ceivers are persistent. In the context of $\mathrm{CCP}$, such a restriction would correspond to CCP with universally-quantified persistent ask operations. In the context of calculi for security, persistent receivers can be used to specify protocols where principals are willing to run an unbounded number of times (and persistent messages to model the fact that every message can be remembered by the spy). In fact, the approach of specifying protocols in a persistent setting, with an unbounded number of sessions, has been explored in [3] by using a classic logic Horn clause representation of protocols (rather than a linear logic one).

In this paper, we present our expressiveness study of linearity and persistence in a well-established framework, namely the asynchronous $\pi$-calculus. This way our study (and its applications) benefits from standard and wellinvestigated reasoning techniques and notions of equivalence. Furthermore the linear/persistent features of the above calculi are naturally captured in this framework. Linear messages are represented as asynchronous outputs, and linear receivers as input processes. Persistent messages (and receivers) can simply be specified using the replication operator of the calculus which creates an unbounded number of copies of a given process.

We consider four sub-languages of the asynchronous polyadic $\pi$-calculus, each capturing one of the above sources of linearity/persistence. Namely, the polyadic asynchronous $\pi$-calculus $(\pi)$, the persistent-input $\pi(\mathrm{PI} \pi)$ defined as $\pi$ but inputs must be replicated, persistent-output defined dually, i.e. outputs rather than inputs must be replicated ( $\mathrm{PO} \pi$ ), and finally persistent $\pi$ defined as $\pi$ but with all inputs and outputs replicated $(\mathrm{P} \pi)$. We conduct our study by providing (or proving the non-existence of) encodings among the fragments, a processes-as-formulae interpretation and a reduction from Minsky machines.

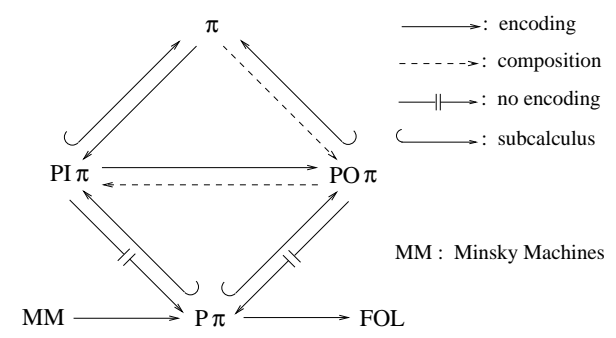

Figure 1. The hierarchy of linearity vs persistence.

Contributions. We provide encodings, homomorphic w.r.t. parallel composition, from $\pi$ into $\mathrm{PI} \pi$ and $\mathrm{PO} \pi$ capturing the behaviour of the source processes. These encodings are, respectively, fully abstract w.r.t. weak barbed congruence and weak barbed congruence restricted to encoded

\begin{tabular}{c|c|c|c|} 
& $\mathrm{P} \pi$ & $\mathrm{PO} \pi$ & $\mathrm{PI} \pi$ \\
\hline 0 & yes & yes & no \\
\hline 1 & $?$ & no & no \\
\hline 2 & no & no & no \\
\hline
\end{tabular}

\section{Table 1. Decidability of barbed congruence for} the $n$-adic ( $n=0,1,2)$ persistent calculi.

contexts. In contrast, we show that it is impossible to provide such encodings from $\pi$ into $\mathrm{P} \pi$. Intuitively this means that we need one source of linearity, i.e. either on inputs $(\mathrm{PI} \pi)$ or outputs $(\mathrm{PO} \pi)$ to capture the behaviour of arbitrary $\pi$ processes via full-abstraction. Despite the impossibility result for $\mathrm{P} \pi$ we also prove that $\mathrm{P} \pi$ is in fact Turingpowerful by encoding Minsky machines. Figure 1 illustrates these expressiveness results (a dashed arrow means that the encoding is obtained via composition).

Furthermore, we consider sub-languages of the above $\pi$-calculi with restricted arity (i.e., the maximum number of names that can be sent in a single communication) and classify them according to the decidability of barbed congruence and their arity. Interestingly, we state that barbed congruence is undecidable for the zero-adic version of $\mathrm{PI} \pi$, the monadic version of $\mathrm{PO} \pi$ and the bi-adic version of $\mathrm{P} \pi$. We also show that barbed congruence is decidable for the zero-adic versions of $\mathrm{P} \pi$ and $\mathrm{PI} \pi$. We leave open the corresponding decidability question for the monadic version of $\mathrm{P} \pi$. Table 1 summarizes these decidability results.

We also show that $\mathrm{P} \pi$ admits a processes-as-formulae compositional interpretation, building on the translation of $\pi$ to linear logic in $[13,23]$ and the logical characterization of CCP languages [11,12]. Specifically, we characterize the standard $\pi$-calculus notion of barbed observability (for $\mathrm{P} \pi$ ) as entailment in First-Order Logic (FOL). Indeed, $\mathrm{P} \pi$ can be seen as a CCP language over the Gentzen constraint system (without function symbols), with persistent universal asks [22]. Furthermore, we exploit classic FOL results by Bernays, Schönfinkel and Gödel to identify classes of infinite-state processes with meaningful mobile behaviour for which barbed reachability is decidable.

Our expressiveness results bear witness to the generality of the persistent store assumption in CCP and calculi for security. Moreover, the processes-as-formulae interpretation of $\mathrm{PO} \pi$ has interesting applications. In particular, the decidability results for barbed reachability for $\mathrm{P} \pi$ may be beneficial for analyzing protocols in which principals (represented as replicated input processes) are willing to run unboundedly many times, as those studied in [3] .

Due to space limitations most proofs will be omitted. They can be found in the extended version of this paper [18]. 


$$
\begin{aligned}
& \text { COM: } \quad \bar{x}\langle\vec{z}\rangle \mid x(\vec{y}) \cdot P \longrightarrow P\{\vec{z} / \vec{y}\} \quad \text { if }|\vec{z}|=|\vec{y}| \\
& \text { PAR: } \frac{P \longrightarrow P^{\prime}}{P\left|Q \longrightarrow P^{\prime}\right| Q} \quad \text { RES: } \frac{P \longrightarrow P^{\prime}}{(\nu x) P \longrightarrow(\nu x) P^{\prime}} \\
& \text { STRUCT: } \frac{P \equiv P^{\prime} \longrightarrow Q^{\prime} \equiv Q}{P \longrightarrow Q}
\end{aligned}
$$

Table 2. Reduction Rules.

\section{The Calculi}

Here we define the calculi we study. We first recall the (polyadic) asynchronous $\pi$-calculus here referred to as $\pi$. The other calculi are defined as syntactic restrictions of $\pi$.

\subsection{Asynchronous Pi Calculus: $\pi$}

We presuppose a countable set of names, ranged over by $x, y, \ldots$, and for each name $x$, a co-name $\bar{x}$. We use $l, l^{\prime}, \ldots$ to range over names and co-names. We use $\vec{x}$ to denote a finite sequence of names $x_{1} x_{2} \ldots x_{n}$ of size $|\vec{x}|=n$. The $\pi$ processes are given by the following syntax:

$P, Q, \ldots:=0|\bar{x}\langle\vec{z}\rangle| x(\vec{y}) \cdot P|(\nu x) P| P|Q| ! P$

requiring that no name may occur more than once in $\vec{y}$.

Intuitively, an output $\bar{x}\langle\vec{z}\rangle$ represents a particle tagged with a name $x$ indicating that can be received by an input process $x(\vec{y}) . P$ which behaves, upon receiving $\vec{z}$, as $P\{\vec{z} / \vec{y}\}$. Furthermore, $x(\vec{y}) . P$ binds the names $\vec{y}$ in $P$. The other binder is the restriction $(\nu x) P$ which declares a name $x$ private to $P$. The parallel composition $P \mid Q$ means $P$ and $Q$ running in parallel. The replication $! P$ means $P|P| \ldots$, i.e., ! $P$ represents a persistent resource.

We use the standard notations $b n(Q)$ for the bound names in $Q$, and $f n(Q)$ for the free names in $Q$, and write $\left(\nu x_{1} \ldots x_{n}\right) P$ to denote $\left(\nu x_{1}\right) \ldots\left(\nu x_{n}\right) P$. We let $\sigma$ range over non-capturing substitutions of names on processes.

The reduction $\longrightarrow$ is the least binary relation on processes satisfying the rules in Table 2 . We use $\longrightarrow$ * to denote the reflexive, transitive closure of $\longrightarrow$. The reductions are quotiented by the structural congruence relation $\equiv$.

Definition 2.1. Let $\equiv$ be the smallest congruence over processes satisfying $\alpha$-equivalence, the commutative monoid laws for composition with 0 as identity, the replication law $! P \equiv P \mid ! P$, the restriction laws $(\nu x) 0 \equiv$ $0, \quad(\nu x)(\nu y) P \equiv(\nu y)(\nu x) P$ and the extrusion law: $(\nu x)(P \mid Q) \equiv P \mid(\nu x) Q$ if $x \notin f n(P)$.

We conclude the description of $\pi$ by recalling some process equivalences we shall use throughout the paper. First we recall a basic notion of observation in the $\pi$-calculus.
Definition 2.2. Define $P \downarrow_{\bar{x}}$ iff $\exists \vec{z}, \vec{y}, R: \quad P \equiv$ $(\nu \vec{z})(\vec{x}\langle\vec{y}\rangle \mid R)$ and $x$ is not in $\vec{z}$. Similarly, $P \downarrow_{x}$ iff $\exists \vec{z}, \vec{y}, Q, R: P \equiv(\nu \vec{z})(x(\vec{y}) \cdot Q \mid R)$ and $x$ is not $\vec{z}$. Furthermore, $P \Downarrow_{l}$ iff $\exists Q: P \longrightarrow{ }^{*} Q \downarrow_{l}$.

Intuitively, given $l=x(l=\bar{x})$ we say that $l$, a $b a r b$, can be observed at $P$, written $P \downarrow_{l}$, iff $P$ can perform an input (output) on channel $x$. However, in the context of the asynchronous $\pi$-calculus in defining the process equivalences it is standard to restrict the observables to be the output barbs; i.e., barbs of the form $\bar{x}$ [21].

We begin with a basic $\pi$ process equivalence, sometimes referred to as barbed correspondence [17], which equates processes iff they exhibit the same barbs. In what follows we prefer to refer to this equivalence as output equivalence since we only consider output barbs. Recall that a process context $C$ is an expression with a hole [.] such that placing a process in the hole produces a process term.

Definition 2.3 (Output Equivalence, Output Congruence). We say that $P$ and $Q$ are output equivalent, written $P \stackrel{\cong}{\simeq}$ iff for every $x, P \Downarrow_{\bar{x}} \Leftrightarrow Q \Downarrow_{\bar{x}}$. We say that $P$ and $Q$ are output congruent, written $P \simeq Q$, iff for every process context $C, C[P] \stackrel{\circ}{\simeq} C[Q]$.

We now recall the notion of (weak) barbed bisimilarity.

Definition 2.4 (Barbed Bisimilarity, Barbed Congruence). $A$ (weak) barbed bisimulation is a symmetric relation $\mathcal{R}$ satisfying the following: $(P, Q) \in \mathcal{R}$ implies that:

1. $P \longrightarrow P^{\prime}$ then $\exists Q^{\prime}: Q \longrightarrow{ }^{*} Q^{\prime} \wedge\left(P^{\prime}, Q^{\prime}\right) \in \mathcal{R}$.

2. $P \downarrow_{\bar{x}}$ then $Q \Downarrow_{\bar{x}}$.

We say that $P$ and $Q$ are (weak) barbed bisimilar, written $P \dot{\approx} Q$, iff $(P, Q) \in \mathcal{R}$ for some barbed bisimulation $\mathcal{R}$. Furthermore, (weak) barbed congruence $\approx$ is defined as: $P \approx Q$ iff for every process context $C[\cdot], C[P] \dot{\approx} C[Q]$.

\section{2 (Semi) Persistent Subcalculi of $\pi$}

The Persistent-Output Calculus: $\mathrm{PO} \pi$. The persistentoutput calculus $\mathrm{PO} \pi$ arises as from $\pi$ by requiring all outputs to be replicated. In fact for $\mathrm{PO} \pi$ processes, $\longrightarrow$ can equivalently be defined as in Table 2 with COM replaced with the rule below. The new rule reflects the linear-input and persistent-output nature of $\mathrm{PO} \pi$.

$$
! \bar{x}\langle\vec{z}\rangle|x(\vec{y}) \cdot P \longrightarrow ! \bar{x}\langle\vec{z}\rangle| P\{\vec{z} / \vec{y}\} \quad \text { if }|\vec{z}|=|\vec{y}|
$$

The Persistent-Input Calculus: $\mathrm{PI} \pi$. The $\mathrm{PI} \pi$ calculus results from $\pi$ by requiring all input processes to be replicated. The relation $\longrightarrow$ for $\mathrm{PI} \pi$ can be equivalently defined as in Table 2 with COM replaced with the rule

$$
\bar{x}\langle\vec{z}\rangle|! x(\vec{y}) \cdot P \longrightarrow P\{\vec{z} / \vec{y}\} \quad| ! x(\vec{y}) \cdot P \quad \text { if }|\vec{z}|=|\vec{y}|
$$


The Persistent Calculus: $\mathrm{P} \pi$. Finally, we have the persistent calculus $\mathrm{P} \pi$ where output and input processes must be replicated. The relation $\longrightarrow$ for $\mathrm{P} \pi$ can be equivalently defined as in Table 2 with COM replaced with the rule

$$
! \bar{x}\langle\vec{z}\rangle|! x(\vec{y}) \cdot P \longrightarrow P\{\vec{z} / \vec{y}\}| ! \bar{x}\langle\vec{z}\rangle \mid ! x(\vec{y}) \cdot P \text { if }|\vec{z}|=|\vec{y}|
$$

The next proposition reflects the duality of $\mathrm{PI} \pi$ and $\mathrm{PO} \pi$.

Proposition 2.5. The following monotone properties hold:

1. If $P$ is a $\mathrm{PO} \pi$ process, $P \longrightarrow Q$ and $P \downarrow_{\bar{x}}$ then $Q \downarrow_{\bar{x}}$.

2. If $P$ is a $\mathrm{PI} \pi$ process $P \longrightarrow Q$ and $P \downarrow_{x}$ then $Q \downarrow_{x}$.

3. If $P$ is a $\mathrm{P} \pi$ process, $P \longrightarrow Q$ and $P \downarrow_{l}$ then $Q \downarrow_{l}$.

\subsection{Calculi Conventions and their Equivalences}

We will work with fragments of the various calculi in terms of arity.

Definition 2.6. Define the arity of $P, \mathcal{A}(P)$, as $\mathcal{A}(\bar{x}\langle\vec{y}\rangle)=$ $|\vec{y}|, \quad \mathcal{A}(x(\vec{y}) \cdot Q)=\max (|\vec{y}|, \mathcal{A}(Q)), \quad \mathcal{A}(Q \mid R)=$ $\max (\mathcal{A}(Q), \mathcal{A}(R)), \mathcal{A}((\nu x) Q)=\mathcal{A}(! Q)=\mathcal{A}(Q)$.

Given $\Sigma \in\{\pi, \mathrm{PO} \pi, \mathrm{PI} \pi, \mathrm{P} \pi\}$, the $k$-adic (version of) $\Sigma, \Sigma^{k}$ is defined as $\Sigma$ except that its processes have arity less or equal to $k$. We decree that $\Sigma^{\omega}=\Sigma$.

Convention 2.7. Henceforth Calc denote the set of calculi $\left\{\pi^{k}, \mathrm{P} \pi^{k}, \mathrm{PI} \pi^{k}, \mathrm{PO} \pi^{k} \mid k \in \mathbb{N} \cup\{\omega\}\right\}$.

Let us now specialize our equivalences and reduction relation to the various calculi.

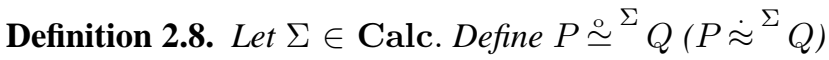
iff $P$ and $Q$ are $\Sigma$ processes and $P \cong Q(P \dot{\sim} Q)$. Also, define $P \simeq^{\Sigma} Q\left(P \approx^{\Sigma} Q\right)$ iff $P$ and $Q$ are $\Sigma$ processes and for every $\Sigma$ context $C, C[P] \stackrel{\circ}{\stackrel{\circ}{ }^{\Sigma}} C[Q]\left(C[P] \dot{\sim}^{\Sigma} C[Q]\right)$. Finally, define $P \longrightarrow_{\Sigma} Q$ iff $P$ and $Q$ are $\Sigma$ processes and $P \longrightarrow Q$.

Notice that reduction, modulo $\equiv$, is invariant wrt the processes of a given calculus $\Sigma \in$ Calc, i.e., if $P \in \Sigma$ and $P \longrightarrow Q$ then there exists $Q^{\prime} \in \Sigma$ such that $Q^{\prime} \equiv Q$.

In what follows when no confusion arises we omit the indices from process relations.

\section{Encodings and their properties}

In the following sections we provide, or under reasonable conditions demonstrate the impossibility of the existence of, encodings $\llbracket \cdot \rrbracket$ from the terms of a given language into the terms of another.

The following condition is particularly appropriate in the context of distributed systems. It describes encodings preserving the parallel topology of the source system.
Definition 3.1. An encoding $\llbracket \cdot \rrbracket$ is a homomorphism w.r.t. parallel composition iff $\llbracket P|Q \rrbracket=\llbracket P \rrbracket| \llbracket Q \rrbracket$. Homomorphism w.r.t the other operators is defined analogously.

The following notions describe some criteria used in the literature for the correctness of encodings (see e.g., [17]).

Definition 3.2. Let $\Sigma, \Sigma^{\prime} \in$ Calc, and $\bowtie \in$ $\{\dot{\sim}, \approx, \stackrel{\cap}{\simeq}, \simeq\}$. Let $\llbracket \cdot \rrbracket: \Sigma \rightarrow \Sigma^{\prime}$ be an encoding (i.e., a map of $\Sigma$ terms into $\Sigma^{\prime}$ terms). The encoding is sound wrt $\bowtie$ iff $\llbracket P \rrbracket \bowtie^{\Sigma^{\prime}} \llbracket Q \rrbracket$ implies $P \bowtie^{\Sigma} Q$. The encoding is complete wrt $\bowtie$ iff $P \bowtie^{\Sigma} Q$ implies $\llbracket P \rrbracket \bowtie^{\Sigma^{\prime}} \llbracket Q \rrbracket$. The encoding is fully abstract wrt $\bowtie$ iff it is both sound and complete wrt $\bowtie$. Finally, $\llbracket \rrbracket$ is ideal wrt $\bowtie$ iff $\llbracket P \rrbracket \bowtie^{\pi} P$.

Intuitively, given a chosen equivalence, full abstraction says that the encoding reflects (soundness) and preserves (completeness) equivalence of source terms. Full abstraction is a useful criterion for the correctness of an encoding wrt a given equivalence when ideal encodings may not exist. Notice that the criterion of being ideal is stronger than that of being fully abstract.

\section{On the Expressiveness of $\mathrm{P} \pi$}

In this section we study the expressiveness of the persistent calculus $\mathrm{P} \pi$. We first prove that it is impossible to provide a sound encoding, homomorphic wrt parallel composition, from $\pi$ into $\mathrm{P} \pi$. This holds for all the equivalences under consideration in this paper-see Definition 3.2.

Despite the above impossibility result, we prove that $\mathrm{P} \pi$ is Turing powerful. We also show $\mathrm{P} \pi$ processes can compositionally be encoded as FOL formulae. We illustrate how mobility in $\mathrm{P} \pi$ can be naturally simulated in FOL and state the characterization of barbed reachability as FOL entailment. We use the characterization and classic FOL theorems to prove decidability results for meaningful classes of infinite-state mobile $\mathrm{P} \pi$ processes.

\subsection{Impossibility of encoding $\pi$ into $\mathrm{P} \pi$}

Key to our impossibility result is establishing the property $P \mid P \approx P$ for arbitrary $\mathrm{P} \pi$ processes $P$. Consider $P=(\nu z) ! \bar{x}\langle z\rangle . P$ may be viewed as a generator of a single private name broadcast on $x$ while $P \mid P$ may be viewed as a generator of two different privates names broadcast on $x$. Therefore, it may not be immediate than $P \mid P$ should be barbed congruent to $P$ in $\mathrm{P} \pi$. In fact, the property would not hold if we had the mismatch operator $[x \neq y] Q$ whose intended meaning is that $Q$ will be executed iff $x$ and $y$ are different names [21], as the following example illustrates:

Example 4.1. Take $R=! x(y) ! x\left(y^{\prime}\right) \cdot\left[y \neq y^{\prime}\right] ! \bar{t}$ and $Q=(\nu z) ! \bar{x}\langle z\rangle$. One can verify that $(R \mid Q) \psi_{\bar{t}}$ but $(R|Q| Q) \Downarrow_{\bar{t}}$. 
The following monotonicity property, which also does not hold in the presence of mismatch, is very useful for our results:

Proposition 4.2. For any name substitution $\sigma, P \longrightarrow Q$ implies $P \sigma \longrightarrow Q \sigma$.

Now, the first interesting result for $\mathrm{P} \pi$ is that output congruence and barbed congruence (restricted to $\mathrm{P} \pi$ contexts) coincide. The result is a corollary of the following lemma whose proof basically rests on showing that $\mathrm{P} \pi$ is confluent.

Lemma 4.3. $\stackrel{\circ}{\simeq}^{\mathrm{P} \pi}=\dot{\sim}^{\mathrm{P} \pi}$.

Corollary 4.4. $\simeq^{\mathrm{P} \pi}=\approx^{\mathrm{P} \pi}$.

We now proceed to prove the Duplication Lemma below. First we need the following Context Lemma whose proof is similar to Lemma 2.1.19 in [21].

Lemma 4.5 (Context Lemma). $P \approx^{\mathrm{P} \pi} Q$ if for every $\mathrm{P} \pi$ process $T$ and name substitution $\sigma$,

$$
T\left|P \sigma \dot{\sim}^{\mathrm{P} \pi} T\right| Q \sigma .
$$

Lemma 4.6 (Duplication Lemma). For every $P \in \mathrm{P} \pi$, $P \mid P \approx{ }^{\mathrm{P} \pi} P$.

Proof. From Corollary 4.3 we can freely replace $\approx$ with $\simeq$. The proof proceeds by induction on the size of $P$. The proof of $P=(\nu x) R$ is particularly interesting and it uses Proposition 4.2 and Lemmas 4.3 and 4.5. The other cases are easier. See [18] for details.

The following proposition can be proven from the above lemmata, and analysis of the reduction of $! P$ in arbitrary contexts.

Proposition 4.7. For every $\mathrm{P} \pi$ process $P, ! P \approx^{\mathrm{P} \pi} P$.

We now have all what we need to prove the following impossibility result.

Theorem 4.8 (Impossibility of Sound Encodings). Let $\bowtie$ $\in\{\dot{\sim}, \approx, \stackrel{\circ}{\simeq}, \simeq\}$. There is no encoding $\llbracket \cdot \rrbracket: \pi \rightarrow \mathrm{P} \pi$, homomorphic wrt parallel composition, such that for all $P, Q \in \pi, \llbracket P \rrbracket \bowtie^{\mathrm{P} \pi} \llbracket Q \rrbracket$ implies $P \bowtie^{\pi} Q$.

Proof. Notice $\stackrel{\circ}{\simeq}^{\pi}$ contains all the other process equivalences of the form $\nwarrow^{\pi}$ while $\approx^{\mathrm{P} \pi}$ is contained in every process equivalence of the from $\bowtie^{\mathrm{P} \pi}$. Then, it suffices to show that there are $P, Q$ such that $\llbracket P \rrbracket \approx{ }^{\mathrm{P} \pi} \llbracket Q \rrbracket$ but $P \gtrless^{\pi} Q$ with $\llbracket \cdot \rrbracket$ being homomorphic wrt parallel composition. Take $P=R \mid R$ and $Q=R$ where $R=\bar{x} \mid x . x . \bar{t}$. Clearly, $P \Varangle^{\pi} Q$ since $P \Downarrow_{\bar{t}}$ but $Q \psi_{\bar{t}}$. From Lemma 4.6 and homomorphism wrt parallel composition we obtain $\llbracket P \rrbracket=\llbracket R \rrbracket \mid \llbracket R \rrbracket \approx^{\mathrm{P} \pi} \llbracket R \rrbracket=\llbracket Q \rrbracket$ as wanted.

\subsection{FOL Characterization of $\mathrm{P} \pi$}

In this section we give a characterization of $\mathrm{P} \pi$ in firstorder logic by providing a compositional translation of $\mathrm{P} \pi$ processes into logical formulae, following the translation of $\pi$ into linear logic [23, Table 2], and the well-known embedding of intuitionistic logic in linear logic through the "of course" modality "!". In particular we shall identify barbed reachability in $\mathrm{P} \pi$ as logical consequence.

We assume the reader is familiar with basic notations and concepts of first-order logic. We presuppose a first-order language $\mathcal{L}$ whose non-logical symbols include predicates of the form out ${ }^{k}$ where $k \geq 0$ denotes the arity of the predicate. We omit this arity index if it is understood from the context. Given two FOL formulae $F$ and $G$ over $\mathcal{L}$, we write $F \models G$ iff the implication formula $(F \Rightarrow G)$ is logically valid. If $F \models G$ we say that $G$ is a logical consequence of $F$.

The following proposition simplifies the kind of $\mathrm{P} \pi$ processes we need to consider in the translation.

Definition 4.9. $A \mathrm{P} \pi$ process is said to be minimal iff it can be generated by the following syntax:

$P, Q, \ldots:=0|! \bar{x}\langle\vec{y}\rangle| ! x(\vec{y}) . P|(\nu x) P| P \mid Q$.

Hence minimal processes are those $\mathrm{P} \pi$ processes where replication only appears immediately before input and output processes. For example, ! $(\nu x) P$ is not minimal.

Definition 4.10. Let $m(\cdot): \mathrm{P} \pi \rightarrow \mathrm{P} \pi$ be the map into minimal processes given as $m(! 0)=m(0)=0, m(! \bar{x}\langle\vec{z}\rangle)=$ $! \bar{x}\langle\vec{z}\rangle, \quad m(! x(\vec{y}) \cdot Q)=! x(\vec{y}) \cdot m(Q), \quad m(!(P \mid Q))=$ $m(P \mid Q)=m(P) \mid m(Q)$ and $m(!(\nu x) P)=$ $m((\nu x) P)=(\nu x) m(P)$.

Proposition 4.11. For every $P \in \mathrm{P} \pi, m(P) \approx^{\mathrm{P} \pi} P$.

Proof. From Proposition 4.7.

Therefore, we can freely restrict ourselves to minimal processes. The following encoding compositionally translates minimal processes into formulae.

Definition 4.12. Let $\llbracket \cdot \rrbracket: \mathrm{P} \pi \rightarrow$ FOL be the partial map from minimal $\mathrm{P} \pi$ terms into $\mathrm{FOL}$ formulae given by:

$$
\begin{aligned}
\llbracket 0 \rrbracket & =\operatorname{true} \\
\llbracket ! \bar{x}\langle\vec{z}\rangle \rrbracket & =\operatorname{out}(x, \vec{z}) \\
\llbracket ! x(\vec{y}) \cdot P \rrbracket & =\forall \vec{y}(\operatorname{out}(x, \vec{y}) \Rightarrow \llbracket P \rrbracket) \\
\llbracket P \mid Q \rrbracket & =\llbracket P \rrbracket \wedge \llbracket Q \rrbracket \\
\llbracket(\nu x) P \rrbracket & =\exists x \llbracket P \rrbracket
\end{aligned}
$$

Intuitively, the above encoding $\llbracket \cdot \rrbracket$ is meant to capture in logic terms how computation proceeds in $\mathrm{P} \pi$. In particular it has the following property: $P$ will perform an output iff 
that output is a logical consequence of $\llbracket P \rrbracket$. Notice that existential quantification corresponds to restriction, which can simulate name extrusion as illustrated below. Also notice that in the translation the two binders of $\mathrm{P} \pi$, input and restriction, are translated into universal and existential quantifiers (resp), hence reflecting an elegant duality.

Example 4.13 (Name Extrusion in FOL). The process $P=$ $(\nu z)(! \bar{x}\langle z\rangle \mid ! z(u) \cdot \bar{u})$ creates a name $z$, broadcasts it to the outside on $x$, and waits on it for a message $u$ from the outside. So, $R=Q \mid P$, with $Q=x(y) . \bar{y}\langle t\rangle$, can perform the output $\bar{t}$, i.e., $R \Downarrow_{\bar{t}}$. Consider the FOL translation in Definition 4.12. We have

$$
\begin{aligned}
\llbracket R \rrbracket= & \forall y(\operatorname{out}(x, y) \Rightarrow \operatorname{out}(y, t)) \\
& \wedge \exists z(\operatorname{out}(x, z) \wedge \forall u \operatorname{out}(z, u) \Rightarrow \operatorname{out}(u)) .
\end{aligned}
$$

which is logically equivalent to

$$
\begin{aligned}
F= & \exists z(\forall y(\operatorname{out}(x, y) \Rightarrow \operatorname{out}(y, t)) \\
& \wedge \operatorname{out}(x, z) \wedge \forall u \operatorname{out}(z, u) \Rightarrow \operatorname{out}(u)) .
\end{aligned}
$$

Now, since out $(z, t)$ is a logical consequence of $\forall y(\operatorname{out}(x, y) \Rightarrow \operatorname{out}(y, t)) \wedge \operatorname{out}(x, z), \quad$ we have $F \quad \models \exists z \operatorname{out}(z, t) \wedge \forall u \operatorname{out}(z, u) \Rightarrow \operatorname{out}(u) \quad$ from which we obtain $F \models$ out $(t)$.

Roughly speaking, the logical step to (4.13) corresponds to using the Structural Equivalence to move a restriction to outermost position (Definition 2.1). The other steps involve Modus Ponens which corresponds to applying rule COM.

The lemma below, which can be proven using induction on length of the derivation, states that $\mathrm{P} \pi$ reduction corresponds to logical consequence.

Lemma 4.14. If $P \longrightarrow_{\mathrm{P} \pi} Q$ then $\llbracket P \rrbracket \models \llbracket Q \rrbracket$.

The following theorem states the characterization of barbed observability in terms of logical consequence. It is related to a similar characterization in [23, Theorem 2.6]. Recall that $\mathcal{A}(P)$ denotes the arity of $P$ (see Definition 2.6) and that from Proposition 4.11, up-to barbed congruence for $\mathrm{P} \pi$, we can confine our attention to minimal processes.

Theorem 4.15 (FOL Characterization of Barbs). Let $\llbracket \cdot \rrbracket:$ $\mathrm{P} \pi \rightarrow$ FOL be the map in Definition 4.12. Let $P$ be a minimal $\mathrm{P} \pi$ process. Then

$$
P \Downarrow_{\bar{x}} \quad \text { if and only if } \llbracket P \rrbracket \models \exists \vec{z} \operatorname{out}(x, \vec{z})
$$

for some $\vec{z}$ such that $|\vec{z}| \leq \mathcal{A}(P)$.

Proof. The proof of the "only-if" direction uses Lemma 4.14. The proof of the "if" direction, the most difficult case, uses a normal form representation of the target formulae. Such normal forms simplifies the analysis of how the formulae on the right-hand of $=$ could have been deduced from $\llbracket P \rrbracket$. See [18].
Remark 4.16. Theorem 4.15 reveals an interesting correspondence between restriction and existential quantification. Intuitively, it holds because we do not have operators than can make use of the fact that two names are different. It would not hold if we had mismatch with the natural translation $\llbracket[x \neq y] P \rrbracket=(x \neq y) \Rightarrow \llbracket P \rrbracket$. E.g., $(\nu x y)[x \neq y] \bar{t}$ can perform $\bar{t}$ while from $\exists x y(x \neq y) \Rightarrow$ out $(t)$ we cannot conclude out $(t)$. For more on this issue see [18].

Applications. The following results are meant to illustrate applications of our FOL characterization. We use classic results from FOL to prove decidability results for barb reachability and barbed congruence.

Decidable $\mathrm{P} \pi$ Classes. The following lemma identifies several classes of $\mathrm{P} \pi$ processes whose barb-reachability problem is decidable. These classes include classes of infinite-state processes with name mobility (extrusion). The reachability question is relevant for safety properties stating that a given undesired output will never be performed.

Lemma 4.17. Let $\llbracket \cdot \rrbracket: \mathrm{P} \pi \rightarrow$ FOL be the map in Definition 4.12. Given $P$ and $z$, the question whether $P \Downarrow_{z}$ is decidable if $P$ belongs to one of the following classes:

1. $\{R \mid \llbracket R \rrbracket \Leftrightarrow \forall \vec{x} \exists \vec{y} F\}$ (Bernays-Schönfinkel's class).

2. $\{R \mid \llbracket R \rrbracket \Leftrightarrow \forall \vec{x} \exists u w \forall \vec{y} F\}$ (Gödel's class).

3. $\left\{R \mid R \equiv R^{\prime}\right.$ for some $R^{\prime}$ s.t. $\left.\left|f n\left(R^{\prime}\right) \cup b n\left(R^{\prime}\right)\right| \leq 2\right\}$ (Two-Variables Class).

4. $\mathrm{P} \pi^{0}$ (Persistent CCS-like Class).

where $F$ is a quantifier-free formula.

Proof. By reducing the question, with the help of Proposition 4.11 , Theorem 4.15 , to the validity of a formula which is in the class of either Bernays-Schönfinkel, Gödel, twovariables, or Monadic FOL formulae without function symbols. All these classes of formulae are decidable [5]. See [18] for details.

Decidable Classes with Mobility. Let us illustrate briefly the name extrusion capabilities of the "mobile" classes in the above lemma. Recall that input and restriction binders are translated into universal and existential quantifiers, respectively. Hence an alternation of inputs and restrictions corresponds to alternation of universal and existential quantifiers. For instance, take $Q=! x(y) \cdot(\nu z) ! \bar{y}\langle z\rangle$. Notice that $Q$ has an input-restriction alternation and $\llbracket Q \rrbracket$ has an universal-existential alternation as it is equivalent to $\forall y \exists z(\operatorname{out}(x, y) \Rightarrow \operatorname{out}(y, z))$.

Consequently, Bernays-Schönfinkel's class allows providers of new names like the process $Q$ above, i.e, processes that upon request, say on a channel $x$, can output private names in a given return channel $y$. It is worth pointing out that this class is closed under parallel composition. 
So $Q$ composed with a process $R$ in the class, remains in the class: e.g. $R$ could be a process $! \bar{x}\langle r\rangle \mid ! r(z) \cdot Q^{\prime}$ requesting from $Q$ a fresh name.

Nevertheless, in general the Bernays-Schönfinkel's class does not allow processes with inputs on private names as the $P=(\nu z)(! \bar{x}\langle z\rangle \mid ! z(u) \cdot ! \bar{u})$ in our name-extrusion example (Example 4.13). However, the Gödel class allows such processes only if the number of such inputs on private names is less than three.

The third class allows processes which can be rewritten (by re-using bound names wherever possible) with only two names. E.g., $P$ and $Q$ above belong to the class since $P \equiv$ $(\nu z)(! \bar{x}\langle z\rangle \mid ! z(x) . ! \bar{x})$ and $Q \equiv ! x(u) \cdot(\nu x) ! \bar{u}\langle x\rangle$.

Decidability Result for Barbed Congruence. It is easy to adapt the results [6] to prove that (weak) barbed congruence is undecidable for the zero-adic version of the $\pi$, in our notation $\pi^{0}$. In contrast, here we prove that (weak) barbed congruence is decidable for the zero-adic version of $\mathrm{P} \pi, \mathrm{P} \pi^{0}$. The proof, which can be found in [18], uses the FOL reasoning.

The following theorem states the decidability of all the equivalences under consideration for $\mathrm{P} \pi^{0}$. It should be noticed that the decidability result for $\simeq$ involves the use of FOL reasoning to characterize a finite set of contexts which is sufficient for verifying the congruence (see [18]).

Theorem 4.18 (Decidable Equivalences of $\mathrm{P} \pi^{0}$ ). Let $\Sigma=$ $\mathrm{P} \pi^{0}$. Given $P, Q$ in $\Sigma$ and $\bowtie \in\{\stackrel{\varrho}{\simeq}, \simeq, \dot{\sim}, \approx\}$, the question whether $P \bowtie^{\Sigma} Q$ is decidable.

\subsection{Turing Expressiveness of $\mathrm{P} \pi$}

In Section 4.1 we proved that there is no sound encoding, homomorphic wrt parallel, from $\pi$ into $\mathrm{P} \pi$. In this section we show that despite such an impossibility result $\mathrm{P} \pi$ is Turing-powerful. We do this by encoding two-counter machines, also called Minsky machines, which are known to be Turing-powerful [16].

Minsky Machines. A two-counter Minsky machine is an imperative program consisting of a sequence of labelled instructions $I_{1} ; \ldots ; I_{k}$ which modify the values of two nonnegative counters $c_{0}$ and $c_{1}$. The instructions, using counters $c_{n}$ for $n \in\{0,1\}$, are of three kinds: $L_{i}$ : halt, $L_{i}: c_{n}:=c_{n}+1 ;$ goto $L_{j}$, and $L_{i}$ : if $c_{n}=$ 0 then goto $L_{j}^{1}$ else $c_{n}:=c_{n}-1$; goto $L_{j}^{2}$. The Minsky machine starts at $L_{s}$ and halts if control reaches the location of a halt instruction. A Minsky machine $M\left(v_{0}, v_{1}\right)$ computes the value $n$ if it halts with $c_{0}=n$.

Encoding Minsky Machines. Our encoding of a given Minsky machine $M$ with start location $L_{s}$ and initial counter values $v_{0}, v_{1}$ into $\mathrm{P} \pi, \llbracket M_{s}\left(v_{0}, v_{1}\right) \rrbracket$, is given below, with the encoding of non-negative numbers in counter $c,[(n)]_{c}$. The counter values are encoded in a standard fashion (similar to the persistent lists in [14]), and each location $L_{i}$ corresponds to a fresh name $l_{i}$ over which the current counter values are passed. Where ever $\overline{l_{j}}\left\langle c, c_{n \oplus 1}\right\rangle$ appears, order the objects correctly based on $n$ ( $\oplus$ denotes addition modulo 2$)$.

$$
\begin{aligned}
& \llbracket M_{s}\left(v_{0}, v_{1}\right) \rrbracket=\left(\nu c_{0}, c_{1}\right)\left(\left[\left(v_{0}\right)\right]_{c_{0}}\left|\left[\left(v_{1}\right)\right]_{c_{1}}\right| ! \overline{l_{s}}\left\langle c_{0}, c_{1}\right\rangle\right. \\
& \left.\mid \prod_{1 \leq i \leq k} \llbracket I_{i} \rrbracket\right) \quad \text { where } M=I_{1} ; \ldots ; I_{k} \\
& \llbracket L_{i}: \text { halt } \rrbracket=! l_{i}\left(c_{0}, c_{1}\right) \cdot ! \overline{h a l t}\left\langle c_{0}\right\rangle \\
& \llbracket L_{i}: c_{n}:=c_{n}+1 ; \text { goto } L_{j} \rrbracket \\
& =! l_{i}\left(c_{0}, c_{1}\right) \cdot(\nu c)\left(S\left(c, c_{n}\right) \mid ! \overline{l_{j}}\left\langle c, c_{n \oplus 1}\right\rangle\right) \\
& \llbracket L_{i}: \text { if } c_{n}=0 \text { then goto } L_{j}^{1} \text { else } c_{n}:=c_{n}-1 \text {; goto } L_{j}^{2} \rrbracket \\
& =! l_{i}\left(c_{0}, c_{1}\right) \cdot(\nu s, z)\left(! \overline{c_{n}}\langle s, z\rangle \mid\right. \\
& \left.\left.[(0)]_{c} \quad=Z(c) \quad=(\nu p)([n-1)]_{j} \mid S(c, p)\right)\left(c_{0}, c_{1}\right\rangle \mid ! s(c) \cdot ! l_{j}^{2}\left\langle c, c_{n \oplus 1}\right\rangle\right) \\
& {[(n)]_{c} \quad=(\nu p)([(n-1)] p \mid S(c, p)) \text { for } n>0} \\
& Z(c) \quad=! c(s, z) . ! \bar{z} \quad \text { (zero) } \\
& S(c, p) \quad=! c(s, z) . ! \bar{s}\langle p\rangle \quad \text { (successor) }
\end{aligned}
$$

In the encoding, because of the persistent nature of $\mathrm{P} \pi$, all states which have been triggered can always be "reexecuted". The encoding of (persistent) counter values uses private channels for signalling successor and zero values, and incremented values are created at private locations. Thus the operations on the counters in one state have no effect on the values encoded in another state - the encoding is free of side-effects.

For example, consider the encoding of the if-then-else instruction. The counter values at the previous enabled location are received over $l_{i}$; the counter $c_{n}$ is asked for its value and will respond on one of the fresh names $s$ and $z$. If it responds on $z$, location $l_{j}^{1}$ is triggered with the current counter values; if it responds on $s$, indicating it is a successor value, then location $l_{j}^{2}$ is triggered with the predecessor of $c_{n}$ (which is received over $s$ ) and the other counter value.

Theorem 4.19. A Minsky machine $M_{s}\left(v_{0}, v_{1}\right)$ computes the value $n$ iff $\llbracket M_{s}\left(v_{0}, v_{1}\right) \rrbracket \mid !$ halt $(c)$.Dec ${ }_{n}(c) \Downarrow \overline{\text { yes }}$ where

$\operatorname{Dec}_{0}(c)=(\nu s, z)(! \bar{c}\langle s, z\rangle \mid ! z . ! \overline{y e s})$

$\operatorname{Dec}_{i}(c)=(\nu s, z)\left(! \bar{c}\langle s, z\rangle \mid ! s(p) . \operatorname{Dec}_{i-1}(p)\right)$ for $i>0$

Applications. In the previous sections we proved that all equivalences and barb reachability problems are decidable for $\mathrm{P} \pi^{0}$. Here we state, on the contrary, that all these problems are undecidable for $\mathrm{P} \pi$.

As a direct consequence of the encoding of two-counter machines, we get undecidability of barbed reachability:

Lemma 4.20. Given $P$ in $\mathrm{P} \pi$ and a name $x$, the question whether $P \Downarrow_{\bar{x}}$ is undecidable.

From the above lemma and a series of reductions (see [18]) we get the following results.

Theorem 4.21 (Undecidable Equivalences of $\mathrm{P} \pi$ ). Let $\Sigma=$ $\mathrm{P} \pi$. Given $P, Q$ in $\Sigma$ and $\bowtie \in\{\stackrel{\circ}{\sim}, \simeq, \dot{\sim}, \approx\}$, the question whether $P \bowtie^{\Sigma} Q$ is undecidable. 
Remark 4.22. In fact, the above undecidability results (Lemma 4.20 and Theorem 4.21) apply already to $\mathrm{P} \pi^{2}-$ they are all obtained from reductions of the Halting Problem for Minsky Machines which were encoded using only the $\mathrm{P} \pi^{2}$ fragment of $\mathrm{P} \pi$. We leave open the corresponding decidability questions for $\mathrm{P} \pi^{1}$-recall that all the corresponding questions are decidable for $\mathrm{P} \pi^{0}$ (Theorem 4.18).

\section{Expressiveness of Semi-Persistent Calculi}

Here we study the expressiveness of the semi-persistent calculi $\mathrm{PI} \pi$ and $\mathrm{PO} \pi$ by means of encodings from $\pi$.

Encoding Linearity. Consider the $\pi$ system:

$$
S=\bar{x}\langle u\rangle|\bar{x}\langle w\rangle| x(y) \cdot \bar{y}\langle m\rangle \mid x(y) \cdot \bar{y}\langle n\rangle
$$

An encoding from $\pi$ into a semi-persistent calculus will be a homomorphism that on $S$ takes the form

$$
\llbracket S \rrbracket=\llbracket \bar{x}\langle u\rangle \rrbracket|\llbracket \bar{x}\langle w\rangle \rrbracket| \llbracket x(y) \cdot \bar{y}\langle m\rangle \rrbracket \mid \llbracket x(y) \cdot \bar{y}\langle n\rangle \rrbracket
$$

Intuitively, to capture the linear communication nature of $\pi$, the encoding would evolve into a process that behaves either as (a) $\llbracket \bar{u}\langle m\rangle \rrbracket \mid \llbracket \bar{w}\langle n\rangle \rrbracket$ or as (b) $\llbracket \bar{w}\langle m\rangle \rrbracket \mid \llbracket \bar{u}\langle n\rangle \rrbracket$. Notice that in each case an output and input (prefix) are consumed.

The obvious problem is that in the semi-persistent calculi either input or outputs are persistent. Let us first discuss the encoding of $\pi$ into $\mathrm{PO} \pi$.

From $\pi$ into $\mathrm{PO} \pi$. A convenient approach is to view (the encoding of) input processes as agents competing for the data of (the encoding of) an output which must become unavailable upon being received by the successful agent.

A naive solution is to have the above-mentioned competing agents send a private channel $r$ on which the output data would be received; e.g., $\llbracket x(\vec{y}) . P \rrbracket=$ $(\nu r)(! \bar{x}\langle r\rangle \mid r(\vec{y}) . \llbracket P \rrbracket)$. The encoded outputs must wait for the private channel on which they send their data; e.g., $\llbracket \bar{x}\langle\vec{z}\rangle \rrbracket=x(r) . ! \bar{r}\langle\vec{z}\rangle$. Now, a problem is then that, e.g., the two encoded outputs in (2) may get the private channel of only one of the encoded inputs, thus making it impossible for the other encoded input to get $u$ or $w$. So one of the encoded outputs will be consumed and the other will be unable to react with other encoded inputs.

The above observation suggests that encoded outputs should also send a secret channel $s$ on which they get a encoded input's secret channel. We could then try

$$
\begin{aligned}
\llbracket \bar{x}\langle\vec{z}\rangle \rrbracket & =(\nu s)(! \bar{x}\langle s\rangle \mid s(r) \cdot ! \bar{r}\langle\vec{z}\rangle) \\
\llbracket x(\vec{y}) \cdot P \rrbracket & =x(s) \cdot(\nu r)(!(\bar{s}\langle r\rangle \mid r(\vec{y}) \cdot \llbracket P \rrbracket)
\end{aligned}
$$

with $\llbracket 0 \rrbracket=0$ and $\llbracket \cdot \rrbracket$ being homomorphic w.r.t all other operators. This solves the above problem, but it creates the dual one. E.g., one of the two encoded inputs in (2) will successfully get the data but the other will be unable to react with another encoded output. It would then seem that we need a more involved protocol to solve the problem.

From $\pi$ to $\mathrm{PI} \pi$ and from $\mathrm{PI} \pi$ to $\mathrm{PO} \pi$. The abovementioned problem of an encoded input being unable to react with other encoded outputs would disappear if such an input was replicated; i.e., if a copy becomes unable to react, we can always try another one. Recall that inputs are always replicated in $\mathrm{PI} \pi$. Thus, an encoding of $\pi$ into $\mathrm{PO} \pi$ may arise from an encoding of $\pi$ into $\mathrm{PI} \pi$ composed with the encoding in (3). Let us then give the latter encoding first.

\subsection{Encoding $\pi$ into $\mathrm{PI} \pi$ : Forwarders}

To make a replicated-input behave as a resource that provides a service only once one may suggest: $\llbracket x(\vec{y}) \cdot P \rrbracket=$ $(\nu l)(\bar{l} \mid ! x(\vec{y}) . ! l \cdot \llbracket P \rrbracket)$ and $\llbracket \bar{x}\langle\vec{z}\rangle \rrbracket=\bar{x}\langle\vec{z}\rangle$. The idea is that the encoded input has a private "lock" $l$ which is activated only once. So, even if the input is replicated, its continuation can be executed only once. Unfortunately the prefix $! x(\vec{y})$ may act as a "sink" consuming several outputs. Nevertheless, a suitable combination of this "lock" idea with a forwarding mechanism leads us to the following encoding:

Definition 5.1. The encoding $\llbracket \cdot \rrbracket: \pi \rightarrow \mathrm{PI} \pi$ is a homomorphism for parallel composition, restriction and replication, otherwise is defined as $\llbracket 0 \rrbracket=0, \llbracket \bar{x}\langle\vec{z}\rangle \rrbracket=\bar{x}\langle\vec{z}\rangle$ and $\llbracket x(\vec{y}) \cdot P \rrbracket=(\nu t f)(\bar{t} \mid ! x(\vec{y}) \cdot(\nu l)(\bar{l}$

where $t, f, l \notin f n(P) \cup\{x, \vec{y}\}$.

$$
\begin{aligned}
& ! t . ! l .(\llbracket P \rrbracket \mid ! \bar{f}) \mid \\
& ! f . ! l . \bar{x}\langle\vec{y}\rangle))
\end{aligned}
$$

Intuitively, an encoded input behaves thus: It creates two flags $t$ and $f$, and then always waits for messages on $x$. The first time it receives a message $\bar{x}\langle z\rangle$, it consumes $\bar{t}$. It then creates the lock $l$-we will comment on the need of this lock below. This way only the !t.!l-branch is activated and the message is accepted by executing $\llbracket P \rrbracket\{\vec{z} / \vec{y}\}$ and activating $\bar{f}$. For every subsequent message $\bar{x}\langle u\rangle$ the input gets, only the ! f.!l-branch is opened, and hence $\bar{x}\langle\vec{u}\rangle$ is forwarded.

Notice that if we did not use the lock $l$, then a "dangling" $f$-branch $! f \cdot \bar{x}\langle\vec{z}\rangle$, resulting after having received the first message $\bar{x}\langle\vec{z}\rangle$, could be opened by an $\bar{f}$. This would cause $\bar{x}\langle z\rangle$ to be forwarded but this message must be consumed.

Properties of $\llbracket \cdot \rrbracket: \pi \rightarrow \mathrm{PI} \pi$. Our encoding is ideal w.r.t barbed congruence. The proof uses a fundamental property of asynchronous $\pi$ : Forwarders are barbed congruent to the null process [10]; i.e. ! $x(\vec{y}) \cdot \bar{x}\langle\vec{y}\rangle \approx 0$.

Lemma 5.2. Let $\llbracket \cdot \rrbracket: \pi \rightarrow \mathrm{PI} \pi$ be the encoding in Definition 5.1. For every $P, \llbracket P \rrbracket \approx^{\pi} P$ holds.

From the above lemma, we get full abstraction w.r.t barbed congruence. 
Theorem 5.3. (Full Abstraction) Let $\llbracket \cdot \rrbracket: \pi \rightarrow \mathrm{PI} \pi$ as in Definition 5.1. For every $P, Q: P \approx Q$ iff $\llbracket P \rrbracket \approx{ }^{\mathrm{PI} \pi} \llbracket Q \rrbracket$.

Application. Using the above encoding we can prove that barbed congruence for the zero-adic version of $\mathrm{PI} \pi, \mathrm{PI} \pi^{0}$, is undecidable. This is to be contrasted with the decidability of $\mathrm{P} \pi^{0}$ shown in the previous section. The results follows from the full-abstraction theorem above and the undecidability of barbed congruence for $\pi^{0}$ which can be proven as that of weak bisimilarity for $\pi^{0}$ in [6].

Theorem 5.4. Let $\Sigma=\mathrm{PI} \pi^{0}$. Given $P, Q \in \Sigma$, the question whether $P \approx^{\Sigma} Q$ is undecidable.

\subsection{Encoding $\pi$ into $\mathrm{PO} \pi$ via composition}

We can now use the above encoding of $\pi$ into $\mathrm{PI} \pi$ to get an encoding of $\pi$ into $\mathrm{PO} \pi$ by composing it with the following encoding from $\mathrm{PI} \pi$ into $\mathrm{PO} \pi$.

Definition 5.5. The encoding $f=\llbracket \cdot \rrbracket: \mathrm{PI} \pi \rightarrow \mathrm{PO} \pi$ is a homomorphism for parallel composition, restriction, and replication, otherwise is defined as $\llbracket 0 \rrbracket=0$, and

$$
\begin{aligned}
\llbracket \bar{x}\langle\vec{z}\rangle \rrbracket & =(\nu s)(! \bar{x}\langle s\rangle \mid s(r) . ! \bar{r}\langle\vec{z}\rangle) \\
\llbracket ! x(\vec{y}) \cdot P \rrbracket & =! x(s) \cdot(\nu r)(! \bar{s}\langle r\rangle \mid r(\vec{y}) \cdot \llbracket P \rrbracket)
\end{aligned}
$$

where $s, r \notin f n(P) \cup\{x, z\}$. Let $g$ be $\llbracket \cdot \rrbracket: \pi \rightarrow \mathrm{PI} \pi$ in Definition 5.1. The encoding $\llbracket \cdot \rrbracket: \pi \rightarrow \mathrm{PO} \pi$ is the composite function $f \circ g$.

Properties of $\llbracket \cdot \rrbracket: \pi \rightarrow \mathrm{PO} \pi$. Let us state the main properties of $\llbracket \cdot \rrbracket: \pi \rightarrow \mathrm{PO} \pi$ given in Definition 5.5. Because of this encoding maps a linear output into a replicated one with the same barb, the encoding does not enjoy the property of being ideal wrt barbed congruence. Notice that replicated inputs were not a problem since the standard barb congruence for $\pi$ does not observe inputs barbs. However, the following proposition states that the encoding is fully-abstract w.r.t. (weak) barbed bisimilarity.

Proposition 5.6. For $\llbracket \cdot \llbracket: \pi \rightarrow \mathrm{PO} \pi$ in Definition 5.5 we have: $P \dot{\approx} Q$ iff $\llbracket P \rrbracket \dot{\sim}^{\mathrm{PO} \pi} \llbracket Q \rrbracket$

Nevertheless, due to the compositional definition of $\llbracket \cdot \rrbracket$, we can give a stronger correspondence result which takes into account weak barbed congruence. Assume that $\llbracket \cdot \rrbracket$ : $\pi \rightarrow \mathrm{PO} \pi$ in Definition 5.5 is extended to process contexts: We decree that $\llbracket[\cdot] \rrbracket=[\cdot]$. Define $\llbracket P \rrbracket \approx_{[\cdot]}^{\mathrm{PO} \pi} \llbracket Q \rrbracket$ iff for every $\pi$ context $C, \llbracket C \rrbracket[\llbracket P \rrbracket] \dot{\approx}^{\mathrm{PO} \pi} \llbracket C \rrbracket[\llbracket Q \rrbracket]$.

Theorem 5.7 (Full-Abstraction wrt Encoded Contexts). Let $\llbracket \cdot \rrbracket: \pi \rightarrow \mathrm{PO} \pi$ be the encoding given in Definition 5.5. The following holds: $P \approx Q$ iff $\llbracket P \rrbracket \approx_{[\cdot]}^{\mathrm{PO} \pi} \llbracket Q \rrbracket$
Remark 5.8. Intuitively, $\llbracket \rrbracket: \pi \rightarrow \mathrm{PO} \pi$ in Definition 5.5 simulates an atomic communication (i.e., interaction between an output and input process) with a protocol of finer communications in which each of the participant waits at some stage for a message from another-this is not the case for $\llbracket \cdot \rrbracket: \pi \rightarrow \mathrm{PI} \pi$ in Definition 5.1 since encoded outputs do not wait for any message. Thus one can envisage a malicious context which does not behave according to the protocol. This is the same kind of problem of the encodings of the polyadic into the monadic $\pi$ calculus. In fact, the following construction is a counterexample to full-abstraction w.r.t. barbed congruence: Take $P=x . x .0$ and $Q=x .0 \mid x .0$. Clearly, $P \approx Q$. Let $C_{t}=! \bar{x}\langle n\rangle|! \bar{x}\langle m\rangle| ! n(r t f) . ! m\left(r^{\prime} t^{\prime} f^{\prime}\right) . ! \bar{t}$. Verify that $\left(C_{t} \mid \llbracket Q \rrbracket\right) \Downarrow_{\bar{t}}$ but $\left(C_{t} \mid \llbracket P \rrbracket\right) \Downarrow_{\bar{t}}$. Hence, $\llbracket P \rrbracket \not \varpi \llbracket Q$.

Nevertheless following the work of [19,24], we believe we can provide a type system in order to give a stronger correspondence for the encoding. The type system would allow contexts that may not behave as dictated by the protocol but do not interfere either. However, this is out of the scope of this paper.

Applications. Obviously, the undecidability of barbed congruence for $\mathrm{PO} \pi$ follows from its sub-calculus $\mathrm{P} \pi$. However, we left open the (un)-decidability of $\mathrm{P} \pi^{1}$. As an application, we use the encoding $\llbracket \cdot \rrbracket: \pi \rightarrow \mathrm{PO} \pi$ in Definition 5.5 to prove the next undecidability result (see [18]).

Theorem 5.9. Let $\Sigma=\mathrm{PO} \pi^{1}$. Given $P, Q \in \Sigma$, the question of whether $P \approx^{\Sigma} Q$ is undecidable.

So all in all we have shown undecidability for barbed congruence for $\mathrm{PI} \pi^{0}, \mathrm{PO} \pi^{1}$, and $\mathrm{P} \pi^{2}$. However, in contrast to $\mathrm{PI} \pi^{0}$ and like $\mathrm{P} \pi^{0}$, barbed congruence for $\mathrm{PO} \pi^{0}$ is decidable as shown below.

From $\mathrm{PO} \pi^{0}$ into $\mathrm{P} \pi^{0}$. We conclude this section by proving the decidability of barbed congruence for $\mathrm{PO} \pi^{0}$ by encoding it into its subcalculus $\mathrm{P} \pi^{0}$. This encoding also gives us a FOL characterization for $\mathrm{PO} \pi^{0}$.

Definition 5.10. The encoding $\llbracket \cdot \rrbracket: \mathrm{PO} \pi^{0} \rightarrow \mathrm{P} \pi^{0}$ is a homomorphism for parallel composition, restriction, and replication, otherwise is defined as $\llbracket 0 \rrbracket=0, \llbracket ! \bar{x} \rrbracket=! \bar{x}$ and $\llbracket x . P \rrbracket=! x . \llbracket P \rrbracket$.

Notice that linear inputs are interpreted as replicated ones. The following result states that in the context of $\mathrm{PO} \pi^{0}$ this interpretation is ideal wrt barbed congruence.

Proposition 5.11. Let $\llbracket \cdot \rrbracket: \mathrm{PO} \pi^{0} \rightarrow \mathrm{P} \pi^{0}$ be the encoding in Definition 5.10. We have $\llbracket P \rrbracket \approx^{\mathrm{PO} \pi^{0}} P$.

From the above proposition and the decidability $\approx$ for $\mathrm{P} \pi^{0}$ (Theorem 4.18) we obtain the following:

Corollary 5.12. Let $\Sigma=\mathrm{PO} \pi^{0}$. Given $P, Q \in \Sigma$, the question of whether $P \approx^{\Sigma} Q$ is decidable. 
Furthermore using the above encoding and lemma and the processes-as-formulae FOL interpretation of $\mathrm{P} \pi$ (Definition 4.12) we conclude that $\mathrm{PO} \pi^{0}$ can be interpreted likewise. E.g., $x . P$ and $(\nu x) P$ are compositionally interpreted as the formulae out $(x) \Rightarrow \llbracket P \rrbracket$ and $\exists x \llbracket P \rrbracket$, respectively, where $\llbracket P \rrbracket$ is the interpretation of $P$.

Corollary 5.13. Let $f: \mathrm{P} \pi \rightarrow$ FOL as in Definition 4.12, $g: \mathrm{PO} \pi^{0} \rightarrow \mathrm{P} \pi^{0}$ in Definition 5.10 and $m: \mathrm{P} \pi \rightarrow \mathrm{P} \pi$ in Definition 4.10. Let $P \in \mathrm{PO} \pi^{0}$ and $\llbracket P \rrbracket=(f \circ m \circ g)(P)$ :

$$
P \Downarrow_{\bar{x}} \text { if and only if } \llbracket P \rrbracket \models \operatorname{out}(x) .
$$

\section{Future Work}

We presented an expressiveness study of linearity and persistence of processes. In this study, however, we did not consider the issue of divergence. E.g., the encoding of $\pi$ into $\mathrm{PI} \pi$ introduces divergent computations which are ignored by the equivalence we chose in this paper, i.e., weak barbed bisimilarity. It would be interesting to provide an analogous study in which the discrimination power of divergence is taken into consideration.

The encoding of $\pi$ into $\mathrm{PO} \pi$ here provided is fullyabstract w.r.t. to barbed congruence restricted to encoded contexts. We conjecture that a fully-abstract encoding of $\pi$ into $\mathrm{PO} \pi$ w.r.t to barbed congruence cannot exist. A similar conjecture can be stated about the existence of encodings which simulate an atomic communication with a protocol of finer communications in which each participant waits at some stage for a message from another. In fact several works in the $\pi$-calculus literature use such kind of encodings, thus studying such conjectures could be relevant for the process calculi community.

We have studied the expressiveness of $\mathrm{P} \pi$ by encoding Minsky Machines. A more insightful approach could be to encode the $\lambda$-calculus into $\mathrm{P} \pi$ as witnessed by the extensive work on translations of this canonical calculus of computable functions into the $\pi$-calculus [21].

We also showed that barbed observability for $\mathrm{P} \pi$ can be characterized as FOL entailment. The characterization was fundamental to state positive decidability results for infinite-state $\mathrm{P} \pi$ processes. By building on the translation of $\pi$ to linear logic in $[13,23]$ we should be able to characterize barbed observability for all the $\pi$ sub-calculi here studied as entailment in linear logic. The resulting characterizations may provide us with useful reasoning techniques for the $\pi$-calculus from linear logic.

\section{References}

[1] R. Amadio, D. Lugiez, and V. Vanackere. On the symbolic reduction of processes with cryptographic functions. Theoretical Computer Science, 290, 2003.
[2] E. Best, F. de Boer, and C. Palamidessi. Partial order and sos semantics for linear constraint programs. In Proc. of Coordination'97, volume 1282 of LNCS, 1997.

[3] B. Blanchet. From linear to classical logic by abstract interpretation. Information Processing Letters, 95(5), 2005.

[4] M. Boreale and M. Buscemi. A framework for the analysis of security protocols. In Proc. CONCUR'02, volume 2421 of LNCS, 2002.

[5] E. B orger, E. Gr adel, and Y. Gurevich. The Classical Decision Problem. Springer-Verlag, 1997.

[6] N. Busi, M. Gabbrielli, and G. Zavattaro. Comparing recursion, replication and iteration in process calculi. In Proc. ICALP'04, volume 3142 of LNCS, 2004.

[7] F. Crazzolara and G. Winskel. Events in security protocols. In Proc. CCS 2001. ACM Press, 2001.

[8] F. Fages, P. Ruet, and S. Soliman. Linear concurrent constraint programming: operational and phase semantics. Information and Computation, 2001.

[9] M. Fiore and M. Abadi. Computing symbolic models for verifying cryptographic protocols. In Proc. CSFW-14. IEEE, 2001.

[10] K. Honda and N. Yoshida. On reduction-based process semantics. Theoretical Computer Science, 151(2):437-486, 1995.

[11] P. Lincoln and V. Saraswat. Proofs as concurrent processes: A logical interpretation of concurrent constraint programming. Technical report, Xerox PARC, 1991.

[12] N. P. Mendler, P. Panangaden, P. J. Scott, and R. A. G. Seely. A logical view of concurrent constraint programming. Nordic J. of Computing, 2(2):181-220, 1995.

[13] D. Miller. The pi-calculus as a theory in linear-logic. In Proc. of Workshop on Extensions to Logic Programming, volume 660 of LNCS, 1992.

[14] R. Milner. Communicating and Mobile Systems: the $\pi$ calculus. Cambridge University Press, 1999.

[15] R. Milner, J. Parrow, and D. Walker. A calculus of mobile processes, Part I + II. Information and Computation, 100(1):1-77, 1992.

[16] M. Minsky. Computation: Finite and Infi nite Machines. Prentice-Hall, 1967.

[17] U. Nestmann. What is a 'good' encoding of guarded choice? Information and Computation, 156:287-319, 2000.

[18] C. Palamidessi, V. Saraswat, F. Valencia, and B. Victor. On the expressiveness of linearity and persistence in the asynchronous $\pi$-calculus. Technical report, LIX, Ecole Polytechnique, 2006. http://www.lix.polytechnique.fr/ fvalenci.

[19] P. Quaglia and D. Walker. On encoding $\mathrm{p} \pi$ in $\mathrm{m} \pi$. In Proc. FSTTCS'98, volume 1530 of LNCS, 1998.

[20] D. Sangiorgi. The name discipline of uniform receptiveness. Theoretical Computer Science, 221(1-2):457-493, 1999.

[21] D. Sangiorgi and D. Walker. The $\pi$-calculus: A Theory of Mobile Processes. Cambridge University Press, 2001.

[22] V. Saraswat. Concurrent Constraint Programming. The MIT Press, Cambridge, MA, 1993.

[23] V. Saraswat and P. Lincoln. Higher-order linear concurrent constraint programming. Technical report, Xerox PARC, 1992.

[24] N. Yoshida. Graph types for monadic mobile processes. In Proc. FSTTCS'96, volume 1180 of LNCS, 1996. 